\title{
Condom use in combination with ART can reduce HIV incidence and mortality of PLWHA among MSM: a study from Beijing, China
}

Lili Tao' ${ }^{1}$ Min Liur ${ }^{2}$, Shuming $\mathrm{Li}^{1}$, Jue $\mathrm{Liu}^{2}$ and Ning Wang ${ }^{3}$

\begin{abstract}
Background: Condom use and antiretroviral therapy (ART) are effective means to prevent and control HIV transmission. We aimed to assess the effect of condom use in combination with antiretroviral therapy (ART) on HIV incidence and mortality among men who had sex with men (MSM) in Beijing, China.

Methods: We evaluated the effect of condom use, ART, and the combination of both among people living with HIV/AIDS (PLWHA) of MSM in the Chaoyang District of Beijing using the Asian Epidemic Model (AEM). Evaluation indicators included absolute risk reduction (ARR) and the percentage of relative risk reduction (RRR \%).

Results: HIV incidence and prevalence declined substantially when condoms were used by MSM in Chaoyang from 2003 to 2013. The ARR of HIV incidence was from 0 to $0.91 \%$ and the RRR\% was from 0 to 43.93\%. The ARR of HIV prevalence was from 0 to $3.79 \%$ and the RRR\% was from 0 to $31.53 \%$. The HIV mortality rate decreased substantially (ARR from 0 to $1.75 \%$, and RRR\% from 0 to $40.03 \%$ ) when ART was implemented. When condom use combined with ART was implemented in MSM in 2003-2013, HIV incidence declined substantially (ARR from 0 to $0.99 \%$, and RRR\% from 0 to 46.11\%). HIV prevalence was also reduced with an ARR from 0 to 3.5\%, and an RRR\% from 0 to 29. $88 \%$. The HIV mortality also declined substantially (ARR from $-0.01 \%$ to $1.02 \%$, RRR\% from $-1.44 \%$ to $39.98 \%$ ).
\end{abstract}

Conclusions: Among MSM, a combination of condom use and ART reduces both HIV incidence and mortality caused by HIV. Combining these methods results in a more effective prevention and control of HIV.

Keywords: Condom use, Antiretroviral therapy (ART), Human immunodeficiency virus (HIV)

\section{Background}

High-risk behaviour intervention is one of the most effective means to prevent and control HIV transmission [1]. Condom use is an effective method to prevent the sexual transmission of HIV, the effect of which has been clearly demonstrated in China [2]. Antiretroviral therapy (ART) can effectively decrease the viral load in the blood and body fluids of people living with HIV/AIDS (PLWHA), thereby reducing HIV transmission [3, 4] and mortality. Currently, Pre-Exposure Prophylaxis (PrEP)

\footnotetext{
* Correspondence: liumin@bjmu.edu.cn

${ }^{2}$ Peking University, School of Public Health, No. 38 Xueyuan Road, Haidian District, Beijing, China

Full list of author information is available at the end of the article
}

has been gradually implemented as a preventative measure in populations at high risk of HIV infection [5]. ART was first introduced in 2003 in Chaoyang District, Beijing. With the implementation of the national policy "Four Frees and One Care" and the adjustment of the initial eligibility threshold for therapy, [6] ART coverage increased yearly, with larger numbers of PLWHA benefiting from ART. ART has been shown to prolong the survival of PLWHA and to improve the quality of life [7].

Chaoyang District, located in the northeast of Beijing, covers an area of $470.8 \mathrm{~km}^{2}$. Notably, Chaoyang is the largest urban district of Beijing with a population of $5,050,000$, which is approximately one-fourth of the capital's total population. The first case of HIV infection in Chaoyang was reported in 1990. By December 31, 
2014, a total of 5481 PLWHA cases had been reported in Chaoyang, with male homosexual transmission accounting for $71.2 \%$ of all cases [8]. HIV-positive men who had sex with men (MSM) comprised the largest contingent of HIV infections. In 2003, Chaoyang District implemented the National AIDS Comprehensive Prevention and Control Demonstration Pilot Project. The project focused on peer education, condom use among MSM, and ART among PLWHA. As the project progressed, the rates of condom use and ART coverage improved among MSM in the area.

We used the Asian Epidemic Model (AEM) [9] to evaluate condom use, ART, and the combined effect of both methods on PLWHA who were MSM. The AEM was primarily developed to estimate epidemics and to simulate the spread of HIV in Asian countries [9-11]. With this model, we used the concept of "a CauseDeleted Life Table" in a fixed area [12] and a relatively stable population to estimate the effect of the above methods on HIV incidence and HIV-related deaths of PLWHA.

\section{Methods}

Aim

We aimed to assess the effect of condom use in combination with antiretroviral therapy (ART) on HIV incidence and mortality from 2003 to 2013 among MSM in Beijing.

\section{Study design}

\section{The Asian epidemic model}

The model we used to evaluate the effectiveness of interventions was AEM (version 4.0), which was developed by Dr. Tim Brown et al., with support from the United States Agency for International Development (USAID). AEM is a full process model that is used to estimate and project the spread of HIV and to assess interventions in Asian areas [13]. The model calculates the numbers of new HIV infections in each subgroup based on different routes of transmission. It can also be used to calculate the number of current HIV infections and HIV-related deaths [14].

\section{(1) Calculation of the number of new HIV infections.}

The number of new HIV infections (new HIV cases in one year) was calculated based on sexual frequency, multiplying the probability of HIV infections per dangerous behaviour and corrections for co-infection with HIV transmission.

For example, the number of new sexual infections of clients by direct sex workers was calculated as previously described [10]:

$$
\begin{aligned}
\mathrm{n}= & \mathrm{p}_{\mathrm{f}_{\mathrm{m}}} \mathrm{X}_{1} \mathrm{~V}_{1 \mathrm{a}}(t)\left(1-\mathrm{C}_{1 \mathrm{a}}(t)\right)\left(\frac{\mathrm{Y}_{3 \mathrm{a}}}{\mathrm{X}_{3 a}+\mathrm{Y}_{3 \mathrm{a}}}\right) \times\left[\mathrm{C}_{\text {std_m }} \mathrm{F}_{\text {stda }(t)}\right. \\
& \left.+\left(1-\mathrm{F}_{\text {stda }}(t)\right)\right]+\left[\mathrm{C}_{\mathrm{cc}} \mathrm{F}_{\mathrm{cc}}(t)+\left(1-\mathrm{F}_{\mathrm{cc}}(t)\right)\right]
\end{aligned}
$$

$\mathrm{X}_{1}$ was the number of uninfected clients, $\mathrm{V}_{1 \mathrm{a}}(t)$ was the frequency of contact with sex workers, $C_{1 a}(t)$ was the level of condom use, and $X_{3 a}$ and $Y_{3 a}$ were the number of uninfected and infected direct sex workers. The additional components $\left[\mathrm{C}_{\text {std_m }} \mathrm{F}_{\text {stda }(t)}+\left(1-\mathrm{F}_{\text {stda }}(t)\right)\right]$ and $\left[\mathrm{C}_{\mathrm{cc}} \mathrm{F}_{\mathrm{cc}}(t)+\left(1-\mathrm{F}_{\mathrm{cc}}(t)\right)\right]$ at the end of the expression represented the adjustments to the transmission for STD enhancement of transmission and the influence of circumcision, respectively.

The number of new HIV infections in each subgroup by different routes of transmission was calculated in the same way.

(2) Calculation of the number of current HIV infections (people living with HIV/AIDS), AIDS patients, and HIV-related deaths.

After the number of new HIV infections was calculated in each subgroup for each year, we could determine the numbers of new AIDS- and HIV-related deaths based on the progression of an HIV infection developing into AIDS and resulting in death. We obtained the number of current HIV infections using the cumulative HIV infections minus the sum of new AIDS- and HIV-related deaths.

We obtained the number of new HIV infections, current HIV infections, and HIV-related deaths among MSM during 2003-2013 from the results of the general population in Chaoyang District. We then changed the input on behavioural and ART parameters and ran the AEM program again. Next, we determined annual HIV infections and HIV-related deaths under different levels of ART and condom use. The difference between these two results was taken to represent the effect of behaviour and treatment intervention [9].

\section{Source of data [15]}

We divided the population into the following four subgroups according actual transmission route in Chaoyang: (1) female sex workers (FSW) and clients through heterosexual behaviour; (2) MSM including male sex workers (MSW) through homosexual behaviour; (3) IV drug users (IDU) by sharing syringes; and (4) the general population.

1. Population size: Demographic data were derived from the Statistical Yearbooks (1990 to 2010) of the Chaoyang District of Beijing, China $[16,17]$. 
2. Behavioural parameters: Data related to the trends in behavioural changes, including condom use (2003 to 2013) (Table 1), proportions of needle sharing among IDUs (1999 to 2012), and sexual behaviour among IDUs and sex workers (1999 to 2010), were primarily derived from sentinel surveillance programmes offered by the Beijing Chaoyang District Center for Disease Control and Prevention [8, 18-22].

3. HIV prevalence: Data on HIV prevalence was collected from the relevant population groups including FSW, IDU, and MSM at the sentinel surveillance points (2003 to 2013) in the Chaoyang District of Beijing [23-26] (as shown in Table 1).

4. ART-related parameters: ART coverage data (2003-2013) was obtained from the Report of Beijing Chaoyang District Health Bureau, and other ART-related parameters were obtained from the Beijing Chaoyang District AIDS Comprehensive Prevention Information System in China (2003 to 2013) and other published references (Table 1) [27, 28].

5. Epidemiological parameters: Probabilities of HIV transmission via different routes, including vaginal intercourse transmission from males to females, vaginal intercourse transmission from females to males, transmission from anally insertive partners to receptive partners, transmission from anally receptive partners to insertive partners, and shared needles for intravenous drug injections, were from published references [29-32].

\section{Setting of the study Research hypothesis}

We used AEM to estimate the numbers of new HIV infections, current HIV infections, and HIV-related deaths among MSM in Chaoyang. The estimated results were identified because of the combined intervention of AIDS in Chaoyang in this study.

We divided MSM into different scenarios based on the intervention measures: with comprehensive intervention, without condom use, without ART, and without a combination of both condom use and ART. We analysed the differences between new HIV infections, current HIV infections, and HIV-related deaths.

Determining the effect of comprehensive interventions: we assumed that the estimated numbers of new HIV infections, current HIV infections, and HIV-related deaths among MSM in 2003-2013 based on the AEM were the effect of comprehensive interventions.

Determining the effect of interventions other than condom use: we assumed that the rate of condom use per year remained at the level of 2003 (the rate of condom use per year between MSM and MSM was $42.00 \%$; between MSM and MSW, the rate was 55.00\%). Holding other factors unchanged, we calculated the numbers of new HIV infections, current HIV infections, and HIVrelated deaths among MSM from 2003 to 2013, which we considered to be the effect of other interventions apart from condom use.

Determining the effect of interventions other than ART: we assumed that ART coverage and the CD4 threshold for treatment eligibility among PLWHA of MSM remained the same as the 2003 level (ART

Table 1 ART-related parameters, condom use and parameters of HIV prevalence among key affected population in Chaoyang District in 2003-2013

\begin{tabular}{|c|c|c|c|c|c|c|c|c|}
\hline Year & ART coverage (\%) & $\begin{array}{l}\text { Initiation on } \\
\text { CD4 cell } \\
\text { count }(/ \mu l)\end{array}$ & $\begin{array}{l}\text { HIV prevalence } \\
\text { of FSW (\%) }\end{array}$ & $\begin{array}{l}\text { HIV prevalence } \\
\text { of male IDU (\%) }\end{array}$ & $\begin{array}{l}\text { HIV prevalence } \\
\text { of female } \\
\text { IDU }(\%)\end{array}$ & $\begin{array}{l}\text { HIV prevalence } \\
\text { of MSM (\%) }\end{array}$ & $\begin{array}{l}\text { Condom use } \\
\text { between MSM } \\
\text { and MSM (\%) }\end{array}$ & $\begin{array}{l}\text { Condom use } \\
\text { between MSM } \\
\text { and MSW (\%) }\end{array}$ \\
\hline 2003 & 5.06 & 200 & 0.26 & 8.01 & 7.83 & 1.34 & 42.00 & 55.00 \\
\hline 2004 & 10.91 & 200 & 0.34 & 7.68 & 6.98 & 1.55 & 44.20 & 56.08 \\
\hline 2005 & 13.81 & 200 & 0.50 & 6.82 & 8.00 & 3.23 & 46.10 & 62.10 \\
\hline 2006 & 18.27 & 200 & 0.29 & 6.48 & 6.14 & 4.81 & 48.34 & 62.74 \\
\hline 2007 & 21.36 & 200 & 0.71 & 6.04 & 5.66 & 4.50 & 50.08 & 63.30 \\
\hline 2008 & 20.98 & 350 & 0.62 & 5.86 & 6.45 & 5.40 & 51.12 & 63.78 \\
\hline 2009 & 20.11 & 350 & 0.07 & 6.22 & 6.06 & 6.04 & 51.30 & 64.10 \\
\hline 2010 & 21.71 & 350 & 0.15 & 4.26 & 6.67 & 6.09 & 52.09 & 64.56 \\
\hline 2011 & 24.14 & 350 & 0.20 & 3.65 & 3.00 & 5.60 & 53.48 & 65.24 \\
\hline 2012 & 27.40 & 350 & 0.10 & 3.95 & 2.81 & 6.87 & 54.48 & 66.08 \\
\hline 2013 & 29.72 & 350 & 0.10 & 3.70 & 2.85 & 7.12 & 56.00 & 66.70 \\
\hline
\end{tabular}

ART Antiretroviral therapy, FSW Female sex workers, HIV Human immunodeficiency virus, IDU IV drug user, MSM Men who have sex with men, MSW Male sex worker Source: ART coverage, [33] initiation on CD4 cell count, [34] HIV prevalence of FSW, IDU (male and female) and MSM, condom use between MSM and MSM, condom use between MSM and MSW were from the surveillance points in Chaoyang District of Beijing in China (2003-2013) and research findings [23-26] 
coverage was $5.06 \%$; CD4 cell count $\leq 200$ cells $/ \mu \mathrm{l}$ ). Holding other factors unchanged, we calculated the numbers of new HIV infections, current infections, and HIV-related deaths among MSM in 2003-2013, which we considered to be the effect of other interventions apart from ART.

Determining the effect of interventions other than combined condom use and ART: we assumed that ART coverage, the $\mathrm{CD} 4$ threshold for treatment eligibility among PLWHA of MSM, and the rate of condom use remained at the same level as 2003 (ART coverage was 5.06\%; CD4 cell count $\leq 200$ cells $/ \mu$ l; the rate of condom use between MSM and MSM was $42.00 \%$; the rate of condom use between MSM and MSW was 55.00\%). With other factors held unchanged, we calculated the numbers of new HIV infections, current HIV infections and HIV-related deaths among MSM in 20032013, which we considered to be the effect of other interventions apart from a combination of condom use and ART.

\section{Assessing index}

In this study, we used absolute risk reduction (ARR) and relative risk reduction percentage (RRR\%) to represent the effect of interventions. ARR is the absolute difference between rates of event incidence without and with an intervention. A larger ARR indicates a more effective intervention. The $R R R \%$ is a determination of the decrease in the relative risk of an adverse event (e.g., HIV infection) compared to the risk of an adverse event pre-intervention. Thus, the RRR\% indicates a reduction in the degree of relative risk before and after an intervention.

The "events" in the following formula referred to new HIV infections, current HIV infections and HIV-related death.

Effect of condom use among MSM:

$\mathrm{ARR}=$ Event rates without condom use minus event rates with comprehensive interventions.

\section{Event rates without condom use \\ $R R R=\frac{\text {-event rates with comprehensive interventions }}{E \text { vent rates without condom use }} * 100 \%$}

Effect of ART among PLWHA of MSM:

$\mathrm{ARR}=$ Event rates without $\mathrm{ART}$ minus event rates with comprehensive interventions.

\footnotetext{
Event rates without ART

$R R R=\frac{\text {-event rates with comprehensive interventions }}{\text { Event rates without ART }} * 100 \%$
}

Effect of condoms in combination with ART among MSM:
$\mathrm{ARR}=$ Event rates without condoms in combination with ART minus event rates with comprehensive interventions.

$$
R R R=\frac{\begin{array}{l}
\text { Event rates without condom in combination with ART } \\
\text {-event rates with comprehensive interventions }
\end{array}}{\text { Event rates without condom in combination with ART }} * 100 \%
$$

\section{Modelling process}

In this study, we used AEM to assess the impact of condom use in combination with ART on HIV incidence and mortality among MSM in Beijing.

First, we filled in many key inputs, including population size, behavioural parameters, HIV prevalence, ARTrelated parameters, and epidemiological parameters, which were shown in the "source of data." After running the AEM, we gained much data about the number of new HIV infections, current HIV infections, and HIVrelated deaths among MSM during 2003-2013 in the Chaoyang District of Beijing. This was a result of the status of the HIV epidemic with comprehensive interventions among MSM.

Next, we reset the inputs on the rate of condom use from 2003 to 2013 among MSM (remained at the level of 2003) in AEM and maintained the other inputs unchanged to allow for the analysis of the downstream impact of other interventions apart from condom use.

Then, we made an adjustment to the inputs on the ART coverage and the treatment eligibility from 2003 to 2013 (the same as the 2003 level) and held other inputs unchanged. We ran the AEM once again and obtained the effect of other interventions apart from ART.

Lastly, we readjusted the inputs both on the rate of condom use among MSM and on the ART coverage and the treatment eligibility from 2003 to 2013; the other factors remained unchanged. We obtained the effect of the other interventions apart from a combination of condom use and ART.

\section{Results}

Through model fitting, we found that when condoms were used, HIV incidence decreased substantially among MSM between 2003 and 2013 in Chaoyang, with an ARR from 0 to $0.91 \%$, and an RRR\% from 0 to $43.93 \%$. HIV prevalence also declined with an ARR of $0 \sim 3.79 \%$ and an RRR\% of $0 \sim 31.53 \%$ between 2003 and 2013. HIV mortality showed a rising trend with ARR from $-0.61 \%$ to $0 \%$ and RRR\% from $-1.44 \%$ to $35.71 \%$ (Table 2, Figs. 1 and 2).

HIV incidence declined slightly with ART implementation among PLWHA of MSM in 2003-2013 in Chaoyang District. The ARR was from 0 to $0.04 \%$, with an RRR\% from 0 to $3.23 \%$. HIV prevalence increased slightly, with ARR from $-0.29 \%$ to 0 and RRR\% from 0 


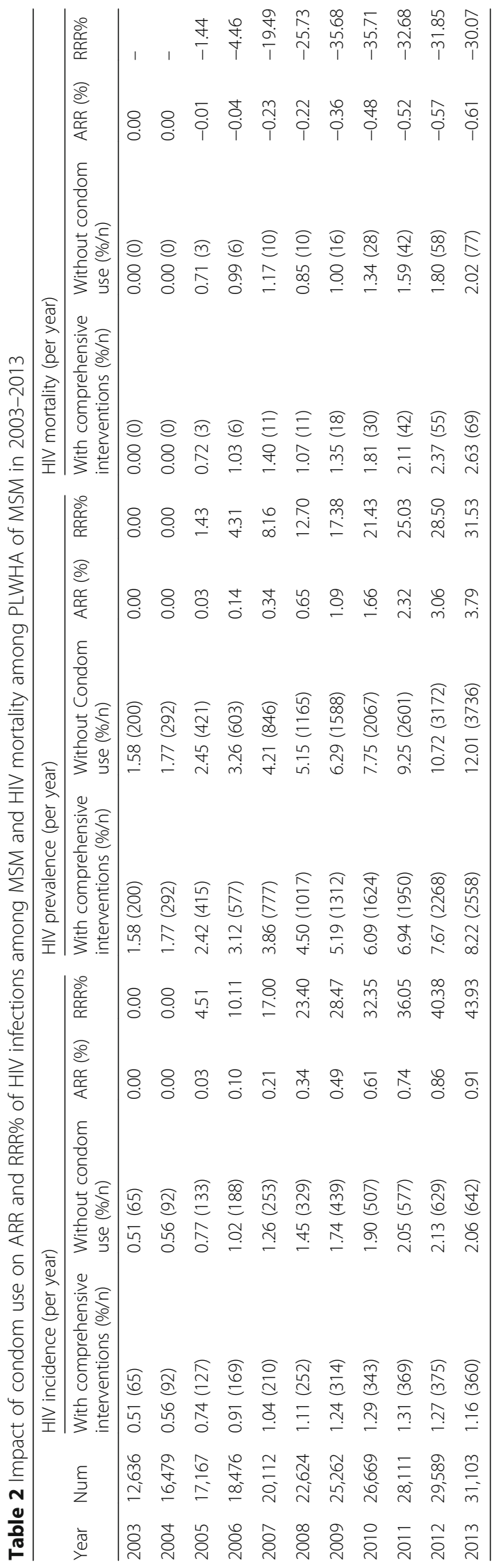




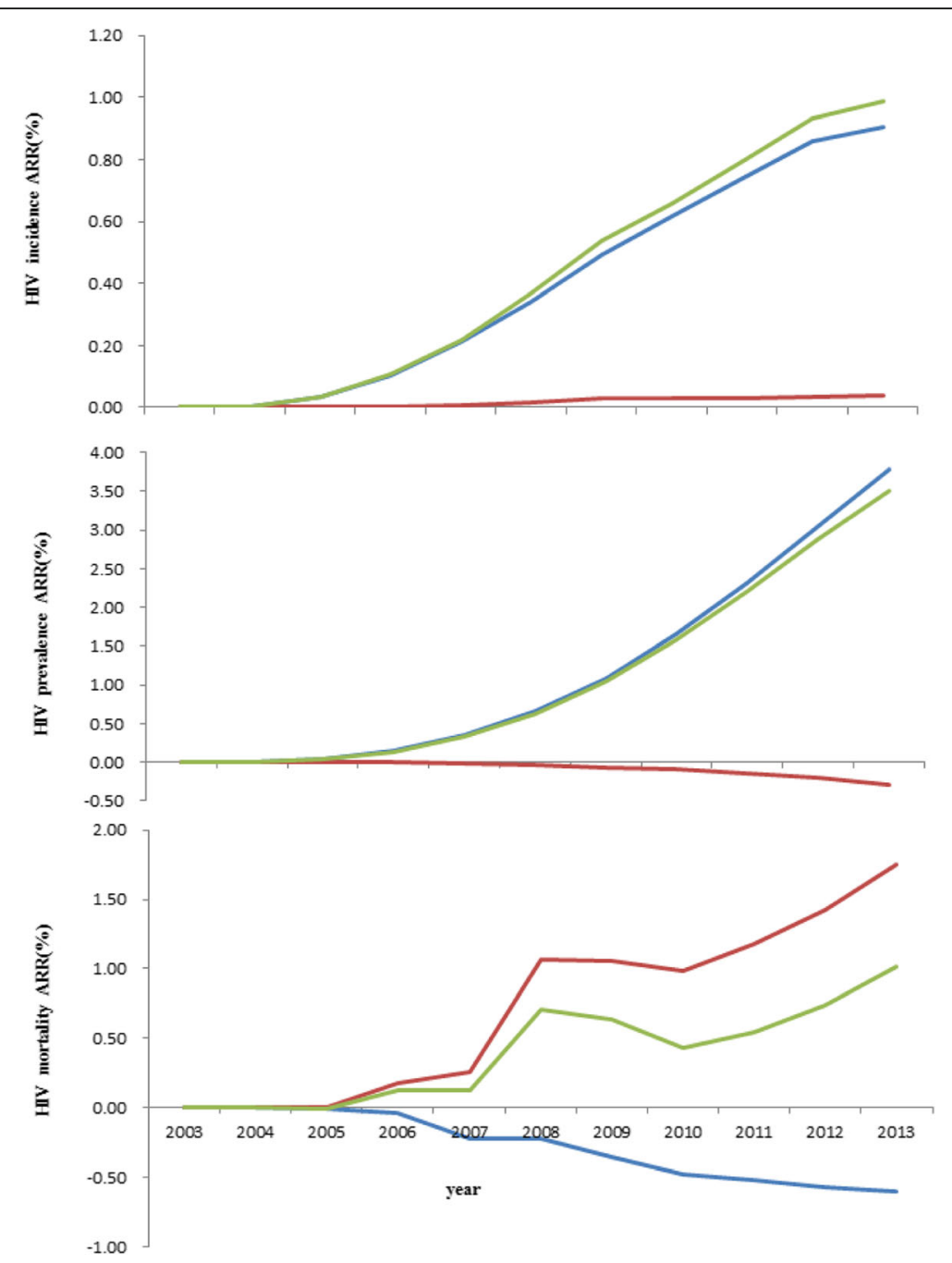

Fig. 1 Impact of condom use, ART and combination of both on ARR of HIV infections among MSM and HIV mortality among PLWHA of MSM in 2003-2013. Condom use, ART, ART combined with condom use

to $3.69 \%$. HIV mortality decreased substantially. The ARR was from 0 to $1.75 \%$ and the RRR\% was from 0 to 40.03\% (as shown in Table 3, Figs. 1 and 2).

HIV incidence declined substantially with a combination of ART and condom use among PLWHA of MSM in 2003-2013. The ARR was from 0 to $0.99 \%$ and the RRR\% was from 0 to $46.11 \%$. HIV incidence decreased slightly with ARR from 0 to $3.5 \%$ and RRR\% from 0 to $29.88 \%$. HIV mortality decreased substantially with ARR from $-0.01 \%$ to $1.02 \%$ and RRR\% from- $1.44 \%$ to $39.98 \%$ (as shown in Table 4, Figs. 1 and 2).

\section{Discussion}

MSM maintain a relatively private status in China. It is challenging to diagnose HIV infections and subsequently carry out interventions aimed at reducing high-risk behaviour among MSM and implementing ART among PLWHA. We have used AEM to evaluate
HIV preventive measures undertaken among MSM in 2003-2013 in Chaoyang, the district with the largest population in Beijing, based on community population statistics. Modelling data was obtained from community demography data of Chaoyang, routine monitoring data of HIV infection status among MSM, special investigations, data from voluntary consultation tests, and published references. This study should provide useful reference data for adjustments of future interventions to prevent the spread of HIV among MSM.

The MSM population has been recognized as the main source of new HIV infections in Chaoyang since 2001. Reducing HIV transmission among MSM can effectively control the progression of the HIV/AIDS epidemic in the district [32]. Condom use and ART were implemented among the MSM population from 2003 to 2013 in the Chaoyang District of Beijing. Compared with 2012, HIV incidence among MSM declined in 2013 and 

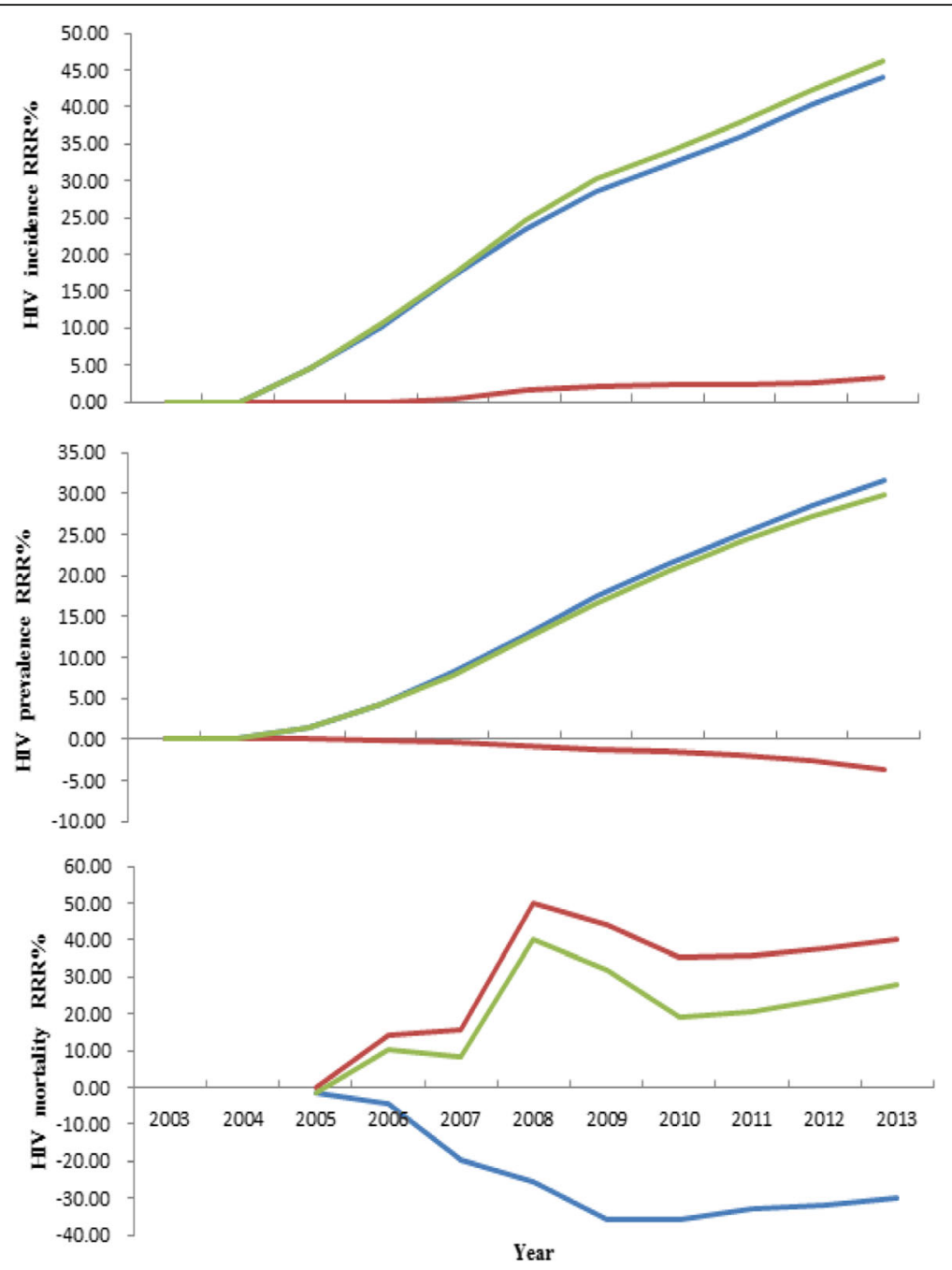

Fig. 2 Impact of condom use, ART and combination of both on RRR\% of HIV infections among MSM and HIV mortality among PLWHA of MSM in 2003-2013. Condom use, -ART, ART combined with condom use

reached the target of the National AIDS Comprehensive Prevention and Control Demonstration Area (HIV incidence rates were reduced by $20 \%-25 \%$ ) [33]. As shown in Table 2 to Table 4, we found that the decline of HIV incidence was estimated to have occurred without condom use and/or ART. One explanation could be that the exposure opportunities of the MSM population decreased due to increasing knowledge and the level of AIDS prevention in high-risk groups, including IDU, FSWs and multiple partners, in addition to the MSM population, along with the improvement of the AIDS prevention level in the whole community.

The rates of condom use between MSM and MSM during sex increased from $42.00 \%$ to $56.00 \%$ in $2003-$ 2013. The rates of condom use also increased from $55.00 \%$ to $66.70 \%$ between MSM and MSW in 20032013. Our current data demonstrate that a simple increase in condom use among MSM resulted in a significant decrease in the relative risk of new HIV infections with an RRR\% of 0 to $43.93 \%$. Condom use also resulted in a decrease in the relative risk of current HIV infections from 0 to $31.53 \%$. This finding is consistent with results from previous studies [34]. However, this study also found that the absolute risk of HIV-related death among PLWHA who are MSM increased slightly from 0 to $0.61 \%$ in $2003-2013$ due to an increase in condom use. The relative risk of HIV-related death also increased slightly from 1.44\% to $35.71 \%$. Possible reasons for the increase in mortality were that the increase in condom use had no direct effect on HIV-related death among PLWHA with a large impact on reducing new HIV infections. It caused the number of new HIV infections to decrease, resulting in an annual reduction in the number of current HIV infections, thus leading to the increase in HIV mortality. 


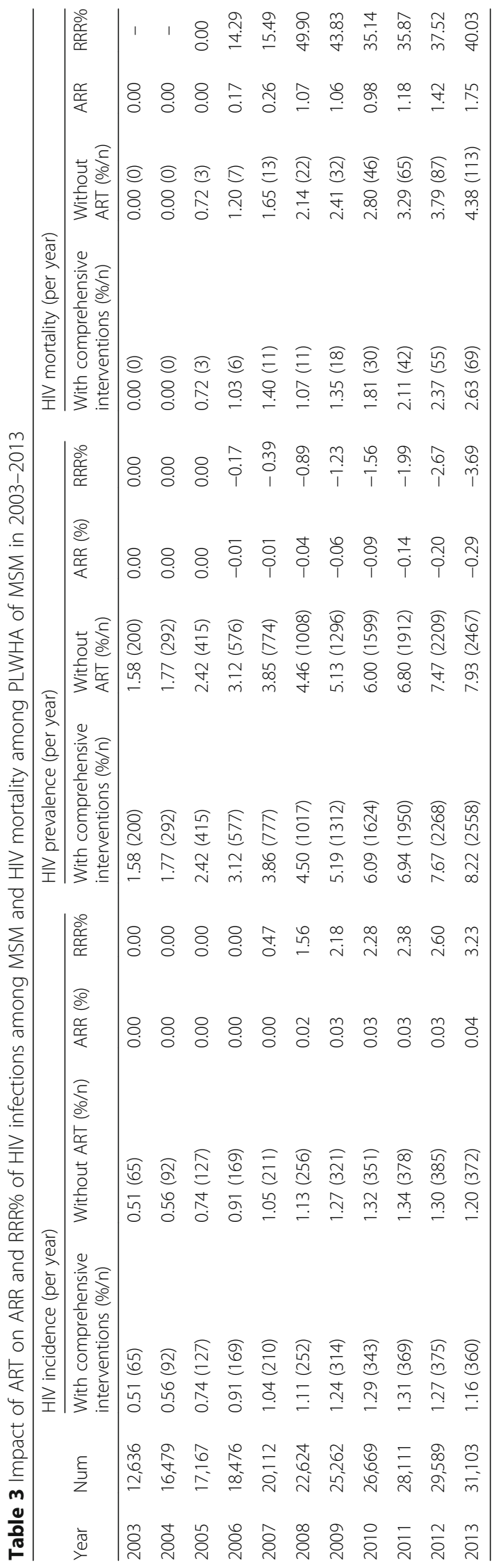




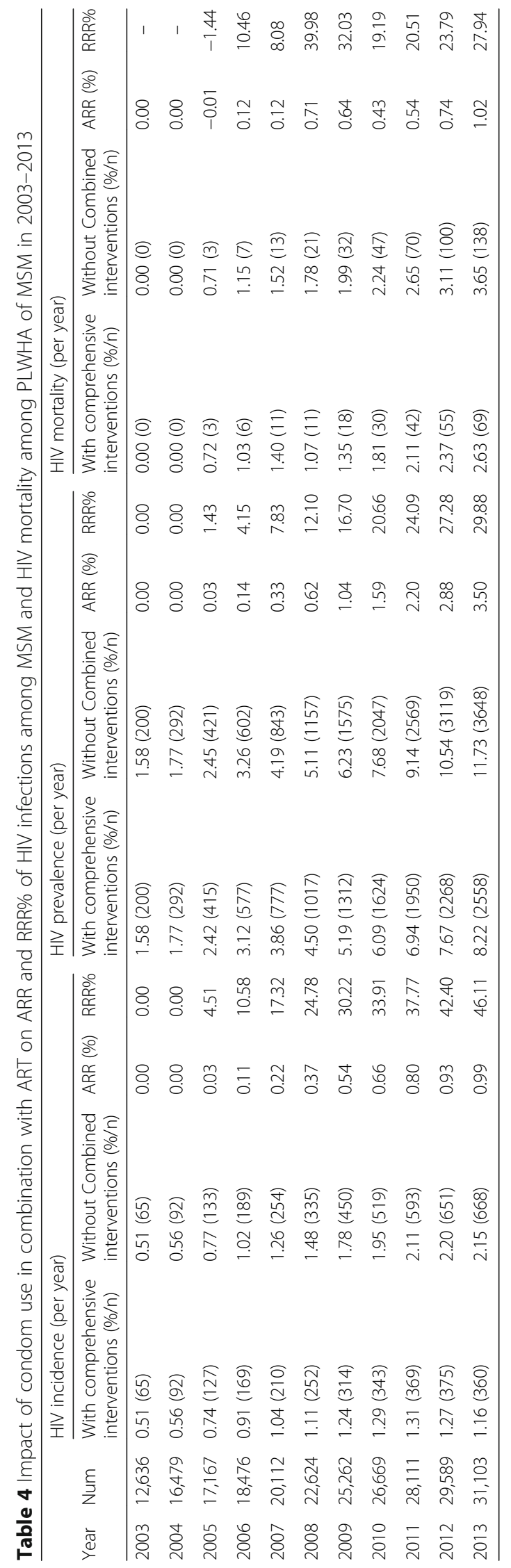


ART was first implemented in 2003 in Chaoyang. ART coverage increased from an initial $5.06 \%$ to $29.72 \%$ in 2013. ART coverage benefited from increased Voluntary Counselling and Testing (VCT) and regular follow-up visits to patients to promote ART treatment among those who qualified for the treatment standard. Our results suggest that HIV incidence among MSM was reduced with the implementation of ART in 2003-2013, which agrees with previous studies [35, 36]. In our study, we found that ART had limited effects in reducing new HIV infections, and the ARR percentage was from 0 to $0.04 \%$ and the RRR percentage was from 0 to $3.23 \%$. It is possible that the effect of ART had not yet been given full attention because the coverage rate of ART was low in 2003-2013 in this area. Additionally, HIV mortality among MSM was dramatically decreased (RRR\% from 0 to $40.03 \%$ ), while both the HIV prevalence and the number of infection sources increased. We found that compared to condom use, the effect of ART on reducing new HIV infections was lower, while the effect of ART in reducing HIV mortality was greater, and HIV mortality clearly decreased after ART implementation.

Our study also found that the effect of condom use in combination with ART on reducing HIV incidence and HIV mortality was greater than the cumulative effect of either ART alone or condom use alone in 2003-2013. Based on the above results, condom use in combination with ART is more effective in preventing the spread of HIV among MSM in Chaoyang. Similar results were reported in the study [37] by Ramadanovic B, which suggested that expanding ART coverage combined with interventions targeting high-risk behaviours amplify the preventive impact.

We admit that this study has several limitations. The AEM is a semi-empirical model, so its effects on the estimation of HIV/AIDS epidemics and the assessment of interventions are restricted by the limitations of the design itself and the experience of the experts involved. In this study, HIV mortality was calculated by HIV deaths/PLWHA/year in AEM, and a more useful measure of the effect of the interventions on HIV mortality would be HIV deaths/100,000 population/year. In addition, this study does not take the influence of heterosexual behaviour of bisexual people among MSM into consideration. The number of heterosexual partners such as FSW and changes in their high-risk behaviour may impact HIV infections among MSM. Moreover, we did not consider drug resistance and ART failure, which may theoretically influence the efficacy of ART.

\section{Conclusions}

In conclusion, interventions aimed at preventing HIV spread among MSM were effective in the Chaoyang District of Beijing in 2003-2013. Condom use can reduce the absolute and relative risk of HIV incidence and
HIV prevalence. ART decreased the absolute risk and relative risk percentage of HIV mortality among PLWHA of MSM. Among MSM, condom use in combination with ART not only reduces HIV incidence but also reduces HIV mortality, and it is more effective in the prevention and control of the spread of HIV.

\section{Abbreviations \\ AEM: Asian Epidemic Model; ARR: Absolute risk reduction; ART: Antiretroviral therapy; FSWs: Female sex workers; IDU: IV drug user; MSM: Men who had sex with men; MSW: Male sex worker; PLWHA: People living with HIV/AIDS; RRR: Relative risk reduction; USAID: United States Agency for International Development}

\section{Acknowledgements}

We thank Jiang Shulin, Wang Lijuan, Wu Wei, Yuan Shengnan, Zhu Junling, Song Liang, Yang Ye, Liu Jun, Hu Yao, and Meng Haiying in the Beijing Chaoyang District Center for Disease Control and Prevention for their research assistance, and Lu Hongyan and Li Guiying in the Beijing Center for Disease Control and Prevention for their advice. We would like to express our thanks to Ma Ning at COFCO Corporation for his comments.

\section{Funding}

This study was supported by the National Key Technology Program from the 12th Five-Year Plan of China (named "the study of projection on the HIV epidemic and mathematical model prediction in China", 2012ZX10001001002). The funding body had no role in the design of the study, the collection, analysis, and interpretation of data or in writing the manuscript.

\section{Availability of data and materials}

The datasets used and/or analysed during the current study are available from the corresponding author on reasonable request.

\section{Ethics approval and consent to participate}

Ethical approval and participant consent were not necessary, as this study involved the use of a previously published de-identified database according to [Ethical review of biomedical research involving human beings], which was promulgated by National Health and Family

Planning Commission of China (http://www.moh.gov.cn/fzs/s3576/ 201610/84b33b81d8e747eaaf048f68b174f829.shtml).

\section{Authors' contributions}

TLL conceived and designed the work that led to the submission and drafted the manuscript. LM revised the manuscript and contributed to model refinement. LSM contributed to data collection. $L$ helped perform the analysis. WN approved the final version. All authors have read and approved the final manuscript.

Consent for publication

Not applicable.

\section{Competing interests}

The authors declare that they have no competing interests.

\section{Publisher's Note}

Springer Nature remains neutral with regard to jurisdictional claims in published maps and institutional affiliations.

\section{Author details}

'Beijing Chaoyang District Centre for Disease Control and Prevention, No. 25 Panjiayuan Huaweili, Chaoyang District, Beijing, China. ${ }^{2}$ Peking University, School of Public Health, No. 38 Xueyuan Road, Haidian District, Beijing, China. ${ }^{3}$ National Center for AIDS/STD Control \& Prevention, No. 155 Chang Bai Road, Changping District, Beijing, China. 
Received: 15 November 2017 Accepted: 28 February 2018 Published online: 13 March 2018

\section{References}

1. Baggaley RF, Garnett GP, Ferguson NM. Modelling the impact of antiretroviral use in resource-poor settings. PLoS Med. 2006;3:e124.

2. Zunyou W. Achievement of HIV AIDS program in the past years and challenges in China. Chinese Journal Of Epidemiology. 2015;36

3. Smith JA, Sharma M, Levin C, Baeten JM, van Rooyen $\mathrm{H}$, Celum C, et al. Cost-effectiveness of community-based strategies to strengthen the continuum of HIV care in rural South Africa: a health economic modelling analysis. Lancet Hiv. 2015;2:e159-68.

4. Rodger AJ, Cambiano V, Bruun T, Vernazza P, Collins S, van Lunzen J, et al. Sexual activity without condoms and risk of HIV transmission in Serodifferent couples when the HIV-positive partner is using suppressive antiretroviral therapy. JAMA. 2016;316:171-81.

5. Harries AD, Lawn SD, Suthar AB, Granich R. Benefits of combined preventive therapy with co-trimoxazole and isoniazid in adults living with HIV: time to consider a fixed-dose, single tablet coformulation. Lancet Infect Dis. 2015;15:1492-6.

6. Zhang Fujie WYYL. Antiretroviral Therapy for HIV/AIDS and Current Situation of China Free ARV Program. Science \& Technology Review 2005,23:24-28.

7. Zhang F, Dou Z, Ma Y, Zhang Y, Zhao Y, Zhao D, et al. Effect of earlier initiation of antiretroviral treatment and increased treatment coverage on HIV-related mortality in China: a national observational cohort study. Lancet Infect Dis. 2011;11:516-24.

8. Song Liang HYJS. Study on HIV and syphilis infections and related risk behaviors among male sex workers in Beijing, China. Chinese J Epidemiol. 2012;33:640-2.

9. Brown T, Peerapatanapokin W. The Asian epidemic model: a process model for exploring HIV policy and programme alternatives in Asia. Sex Transm Infect. 2004;80(Suppl 1):i19-24.

10. Mishra RM, Dube M, Sahu D, Saggurti N, Pandey A. Changing epidemiology of HIV in Mumbai: an application of the Asian epidemic model. Glob J Health Sci. 2012:4:100-12.

11. Ma N, Zheng M, Liu M, et al. Impact of condom use and standardized sexually transmitted disease treatment on HIV prevention among men who have sex with men in Hunan province: using the Asian epidemic model[J]. AIDS Res Hum Retrovir. 2012;28(10):1273-9.

12. Katzmarzyk PT, Lee IM. Sedentary behaviour and life expectancy in the USA: a cause-deleted life table analysis. BMJ Open. 2012;2

13. Cohen J. The Asian epidemic model's provocative curves. Science. 2004;304:1934.

14. Yu Da LMMZ. A process model for exploring the effectiveness of HIV prevention and control policies and programs - an introduction to the Asian epidemic model (AEM). Chin J AIDS STD. 2008;96-97(65):14.

15. Tao LL, Liu M, Li SM, Liu J, Jiang SL, Wang LJ, et al. Impact of antiretroviral therapy on the spread of human immunodeficiency virus in Chaoyang District, Beijing, China: using the Asian epidemic model. Chin Med J. 2017;130:32-8

16. Beijing City TCDB. The fifth census of Beijing Chaoyang District office, Beijing Chaoyang District 2000 census data. In. 2002;

17. Beijing City TCDB. The sixth census of Beijing Chaoyang District office, Beijing Chaoyang District 2010 census data. In. 2012

18. Pan Suiming WWAL. Sexual behavior and sexual relationship in contemporary Chinese. Beijing: Social Sciences Academic Press; 2004

19. Li Dongliang DHLF. An epidemiological analysis of sexual transmitted diseases among 1512 cases of female sex workers. China Journal of Leprosy and Skin Diseases. 2006;22:576-7.

20. THE THAI WORKING GROUP PROJECTIONS. The Asian Epidemic Model (AEM) Projections for HIV/AIDS in Thailand: 2005-2025. In; 2005.

21. Dongliang $L$, Zheng Z, Fengji L. Surveillance analysis of prevalence of sexually transmitted disease in Beijing Chaoyang district from 1993 to 2003. Chinese Journal of AIDS \& STD 2005,11:45-47.

22. Zhaoli Z, Hongyuan L, Ye Y. Survey of factors associated with unprotected sexual behaviors among men who have sex with men in Beijing. Chin J Natural Med. 2008;10:241-5.

23. Yingjie LMYBW. Epidemiology characteristics, sexually transmitted disease and HIV/AIDS status among 403 female sex workers in Chaoyang District, Beijing. CHINESE JOURNAL OF DRUG DEPENDENCE. 2006;5:401-4.
24. Yan-jie G, MYSL. Prevalence and Predictors of HIV, syphilis and herpes simplex type 2 virus ( HSV-2 ) infections among the men who have sex with men ( MSM ) in Beijing. Chin J Public Health. 2012;4:451-3.

25. Dong-liang LIYGMY. Study on the incidence of HIV and associated risk factors through a prospective cohort among men who have sex with men in Beijing, China. Chinese J Epidemiol. 2012;7:663-6.

26. Wei WYLSJ. Achievement of HIV AIDS program in the past years and challenges in China. Chinese J Epidemiol. 2015;12

27. Donnell D, Baeten JM, Kiarie J, Thomas KK, Stevens W, Cohen CR, et al. Heterosexual HIV-1 transmission after initiation of antiretroviral therapy: a prospective cohort analysis. Lancet. 2010;375:2092-8.

28. Cohen MS, Chen YQ, McCauley M, Gamble T, Hosseinipour MC Kumarasamy $\mathrm{N}$, et al. Prevention of HIV-1 infection with early antiretroviral therapy. N Engl J Med. 2011;365:493-505.

29. THE THAI WORKING GROUP ON HIV AIDS PROJECTIONS. The Asian epidemic model (AEM) Projections for HIV/AIDS in Thailand: 2005-2025[R].2005. In.

30. Jin F, Jansson J, Law M, Prestage GP, Zablotska I, Imrie JC, et al. Per-contact probability of HIV transmission in homosexual men in Sydney in the era of HAART. AIDS. 2010;24:907-13.

31. Baggaley RF, White RG, Boily MC. HIV transmission risk through anal intercourse: systematic review, meta-analysis and implications for HIV prevention. Int J Epidemiol. 2010;39:1048-63.

32. Dongliang LI, Yanjie G, Mingrun $Y$, Xueying $Y$, Shuming $L$, Jie $X$, Yingjie L. Study on the incidence of HIV and associated risk factors through a prospective cohort among men who have sex with men in Beijing, China. Chinese J Epidemiol. 2012;33:663-6.

33. Beijing Chaoyang District Health bureau. 12th Five-Year Planning of AIDS prevention and control in Chaoyang District, Beijing. In; 2012

34. AA L, N H MS. Condom distribution in jail to prevent HIV infection. AIDS Behav. 2013;(8):2695-702.

35. Montaner JS, Lima VD, Barrios R, Yip B, Wood E, Kerr T, et al. Association of highly active antiretroviral therapy coverage, population viral load, and yearly new HIV diagnoses in British Columbia, Canada: a population-based study. Lancet. 2010;376:532-9.

36. Shafer LA, Nsubuga RN, Chapman R, O'Brien K, Mayanja BN, White RG. The dual impact of antiretroviral therapy and sexual behaviour changes on HIV epidemiologic trends in Uganda: a modelling study. Sex Transm Infect. 2014;90:423-9.

37. Ramadanovic B, Vasarhelyi K, Nadaf A, Wittenberg RW, Montaner JS, Wood E, Rutherford AR. Changing risk behaviours and the HIV epidemic: a mathematical analysis in the context of treatment as prevention. PLoS One. 2013:8:e62321.

\section{Submit your next manuscript to BioMed Central and we will help you at every step:}

- We accept pre-submission inquiries

- Our selector tool helps you to find the most relevant journal

- We provide round the clock customer support

- Convenient online submission

- Thorough peer review

- Inclusion in PubMed and all major indexing services

- Maximum visibility for your research

Submit your manuscript at www.biomedcentral.com/submit
) Biomed Central 